\title{
Estratégias de Resolução dos Conflitos Conjugais: Percepções de um Grupo Focal ${ }^{1}$
}

\author{
Crístofer Batista da Costa \\ Clarisse Pereira Mosmann \\ Universidade do Vale do Rio dos Sinos \\ São Leopoldo, RS, Brasil
}

\begin{abstract}
RESUMO
A resolução dos conflitos no casamento está associada às estratégias utilizadas pelos cônjuges. Entretanto, os estudos sobre as estratégias e suas repercussões na conjugalidade são escassos no contexto nacional. O objetivo desta pesquisa foi identificar e compreender as estratégias de resolução de conflito utilizadas em casamentos de longa duração e a sua reverberação na dinâmica conjugal. Trata-se de um estudo qualitativo, exploratório e descritivo. Os dados foram coletados por meio de grupo focal, composto por nove pessoas, e submetidos à análise de conteúdo. Foram identificadas 'estratégias de prevenção' dos conflitos auto observando emoções e reações, atentando à predisposição do parceiro ao diálogo e à gravidade dos conflitos. Expectativas e estratégias podem mudar ao longo do tempo de casamento enquanto determinadas características do parceiro e padrões de interação são estáticos e fazem parte da dinâmica do relacionamento que se atualiza e se retroalimenta por meio de questões individuais e conjugais.
\end{abstract}

Palavras-chave: Casamento; conflito conjugal; resolução de problemas.

\begin{abstract}
Marital Conflict Resolution Strategies: Perceptions of a Focus Group

The resolution of conflict in marriage is associated with the strategies used by the spouses. However, the lack of studies on strategies and their impact on marital reveals the need for research in the national context. The objective was to identify and understand the conflict resolution strategies used in long-term marriages and its reverberations in the couple dynamic. This is a qualitative, exploratory and descriptive study. Data were collected through a nine people focus group and subjected to content analysis. From the results were identified conflict 'prevention strategies' self observing emotions and reactions, attention to the predisposition of partner dialogue and severity of conflicts. Expectations and strategies may change long-term in the marriage while some partner's characteristics and interaction patterns are static and are part of marital dynamics that updates and feeds itself through individual and marital issues.
\end{abstract}

Keywords: Marriage; Marital conflict; Problem solving.

\section{RESUMEN}

\section{Estrategias de Resolución de Conflictos de Pareja: Percepciones de un Grupo Focal}

La resolución de conflictos en el matrimonio está asociada a las estrategias utilizadas por los cónyuges. Sin embargo, la falta de estudios sobre las estrategias en el contexto nacional y su impacto en el matrimonio revelan la necesidad de investigación del fenómeno. Se objetivó identificar y comprender las estrategias de resolución de conflicto utilizadas en matrimonios de larga duración y sus reverberaciones en la dinámica conyugal. Se trata de un estudio cualitativo, exploratorio y descriptivo. Los datos fueron recolectados por medio de un grupo focal, con nueve personas, y sometidos al análisis de contenido. Se identifican 'estrategias de prevención' de los conflictos por medio de auto observación de las emociones y reacciones, prestar atención a la predisposición de diálogo de lo cónyuge y la gravedad de los conflictos. Expectativas y estrategias pueden cambiar con el tiempo de matrimonio mientras que ciertas características de algunos cónyuges y los patrones de interacción son estáticos y son parte de la dinámica de pareja la cual se actualiza y retroalimenta a través de cuestiones individuales y matrimoniales.

Palabras-clave: Matrimonio; Conflicto conyugal; Resolución de problemas. 


\section{INTRODUÇÃO}

Os estudos sobre os conflitos conjugais apontam que as variações de uma situação de conflito dentro do casamento dependerão, principalmente, da forma como os cônjuges tentarão resolvê-la (Hoppmann \& Blanchard-Fields, 2011; Leggett, Roberts-Pittman, Byczek, \& Morse, 2012; McNulty, O’Mara, \& Karney, 2008; Paleari, Regalia, \& Fincham, 2010; Schoebi, Karney, \& Bradbury, 2012; Veldorale-Brogan, Lambert, Fincham, \& DeWall, 2013). Na literatura, essa tentativa de resolver os conflitos é chamada de estratégias de resolução, comportamentos, diante das situações conflituosas, por meio dos quais se busca, antecipadamente, solucionar o problema. As estratégias possuem duas características fundamentais: a) definição clara da área de conflito sobre a qual as respostas serão construídas, caso contrário, as estratégias serão inconclusas tanto quanto o problema; e b) desejo mútuo de investir na relação e na resolução dos conflitos (Carlson \& Dinkmeyer, 1987; Leggett et al., 2012; Schoebi et al., 2012).

As estratégias são divididas em construtivas e destrutivas. As construtivas envolvem: compreender as limitações do parceiro e da relação; identificar os aspectos positivos de um conflito; investir na tentativa de resolver os problemas; comunicar de forma respeitosa opiniões e percepções; flexibilizar a negociação de interesses individuais com o parceiro, de modo que ambos tenham suas necessidades atendidas; gerenciar as próprias emoções; entre outras formas (Anderson \& Johnson, 2010; Falcke, Wagner, \& Mosmann, 2013; Rasera \& Guanaes, 2010; Sierau \& Herzberg, 2012; Sullivan, Pasch, Johnson, \& Bradbury, 2010; Wheeler, Updegraff, \& Thayer, 2010; Whiting, 2008).

Por outro lado, algumas tentativas de resolver os conflitos interferem negativamente na situação, caracterizando-se como estratégias destrutivas. Estas envolvem: hostilidade frente às divergências; reclamações; retraimento; silêncio; discussões em tom exaltado sem consenso ou negociação; ofensas; acusações; imediatismo; comportamentos de retirada diante da situação; indisposição à resolução dos conflitos; racionalização e foco excessivo nos interesses pessoais em detrimento dos conjugais (Anderson \& Johnson, 2010; Falcke et al., 2013; Rasera \& Guanaes, 2010; Sierau \& Herzberg, 2012; Smith et al., 2009; Sullivan et al., 2010; Wheeler et al., 2010; Whiting, 2008).

A existência de estratégias construtivas e destrutivas não significa que um casal utilizará apenas uma delas. É esperado que os cônjuges alternem entre essas duas formas durante um episódio de conflito e ao longo do ciclo vital (Falcke et al., 2013; Wheeler et al., 2010). Nesse sentido, quais fatores influenciarão para que as estratégias construtivas de resolver os problemas predominem sobre as destrutivas ou o inverso?

Os estudos têm encontrado diferentes variáveis que interferem na forma como os cônjuges resolvem os seus conflitos. São apontadas: a área de conflito, conjugal ou pessoal (Verhofstadt, Buysse, Ickes, De Clercq, \& Peene, 2005); as características específicas do conflito e da personalidade dos parceiros (Hoppmann \& Blanchard-Fields, 2011); e os efeitos ator e sócio durante as situações conflituosas (Cundiff, Smith, \& Frandsen, 2012). Segundo Cundiff et al., as características de personalidade dos cônjuges produzem o efeito sócio, se os parceiros se influenciam reciprocamente, e o efeito ator, se expectativas e preconcepções interferem na resolução do conflito, funcionando como uma profecia autorrealizadora.

Outros pesquisadores (Madhyastha, Hamaker, \& Gottman, 2011) defendem que a resolução dos conflitos conjugais é determinada pela repercussão das interpretações que o próprio indivíduo faz dos motivos subjacentes ao conflito, semelhante ao efeito ator (Cundiff et al., 2012). Segundo Madhyastha et al. (2011), a resolução dos conflitos é influenciada também pelo que chamaram de inércia emocional, dificuldade de alterar determinados pensamentos e comportamentos por outros, uma vez que mudar exige maior esforço e energia do que permanecer da mesma forma. Para os autores, tais questões seriam mais relevantes do que a influência mútua de positividade e de negatividade oriunda da interação.

Veldorale-Brogan et al. (2013) referem que a resolução dos conflitos é mediada por três fatores: 1) comunicação, capacidade de negociação frente às divergências; 2) virtude, capacidade de perdoar os erros do parceiro e se doar ao relacionamento; e 3) identidade, sentimentos de segurança e de autoestima. Segundo os pesquisadores, a comunicação é perpassada pelos outros dois fatores, isto é, dependerá do quanto se é virtuoso, capaz de perdoar erros, tolerar as limitações do relacionamento e se doar para que ele melhore e do quanto se está seguro e bem consigo mesmo. Além disso, defendem que os comportamentos de apoio durante as situações de conflito, característica dos cônjuges virtuosos, aumentam a intimidade e a proximidade com o parceiro, que se sentirá compreendido em seus desejos, necessidades e valores. E que a percepção dos aspectos positivos no casamento contribui para a resolução construtiva dos conflitos, enquanto enfatizar os problemas repercute negativamente.

Tais pressupostos corroboram as pesquisas sobre as dimensões positiva e negativa do perdão (Fincham, 
Beach, \& Davila, 2004; Fincham, Beach, \& Davila, 2007; Paleari et al., 2010). A benevolência, dimensão positiva, é apontada como estratégia de resolução construtiva; trata-se da capacidade de identificar os pontos fortes do relacionamento e de si mesmo durante os conflitos conjugais. Segundo os autores, a benevolência reverbera também em comportamentos de cooperação e auto revelação entre os parceiros.

Por outro lado, McNulty et al. (2008) salientam que estratégias de benevolência não funcionam ao serem os conflitos conjugais graves e frequentes pois, em longo prazo, estas estratégias repercutem em menores níveis de satisfação conjugal. Nessas situações, seria mais indicado reconhecer e abordar ativamente o problema ao invés de tentar identificar os pontos positivos da relação e de si.

Nesse sentido, atitudes avaliadas em um primeiro momento como negativas, como dar ordens, controlar e tomar decisões sem consultar o parceiro, mediam a resolução de conflitos graves e podem ser preditoras de satisfação conjugal nas relações em que há problemas mais sérios como, por exemplo, uso de substâncias psicoativas. Porém, predizem declínio na satisfação conjugal diante dos problemas menos graves (McNulty et al., 2008; McNulty \& Russell, 2010). Estes apontamentos podem indicar que a reverberação das estratégias de resolução dependerá, também, dos tipos de conflito e do contexto, sendo importante avaliar cada situação antes de inferir um prognóstico da dinâmica conjugal.

Além disso, a dimensão negativa do perdão está associada às estratégias de resolução destrutivas. Tal dimensão envolve comportamentos de retaliação e esquiva e sentimentos de indignação, vingança e mágoa (Fincham et al., 2007; McNulty, et al., 2008; Paleari et al., 2010). Segundo os pesquisadores, se posturas de esquiva e retaliação estiverem presentes, os esforços para a resolução construtiva dos problemas serão desperdiçados. Em outras palavras, é equivocado pensar que o perdão às transgressões do parceiro justifica os comportamentos destrutivos, uma vez que estes comportamentos provocariam repercuções inversas e afetariam negativamente o relacionamento conjugal.

Compreende-se, então, que as estratégias de resolução dos conflitos conjugais estão associadas a diferentes variáveis. Segundo os pesquisadores (Hoppmann \& Blanchard-Fields, 2011; Leggett et al., 2012; Paleari et al., 2010; Tallman \& Hsiao, 2004), a estratégia de cooperação, por exemplo, envolve a participação ativa dos cônjuges, a aceitação de propostas conciliatórias e a adesão à comportamentos efetivos de mudança, que representarão mais esforços a curto prazo, porém, mais benefícios a longo prazo (Schoebi et al., 2012). Ademais, os autores referem que a cooperação entre os cônjuges dependerá também de outros fatores, como a confiança mútua, a empatia e a satisfação conjugal (Paleari et al., 2010; Schoebi et al., 2012; Tallman \& Hsiao, 2004; Verhofstadt et al., 2005).

Têm sido investigados também a utilização dos pronomes pessoais durante a interação conjugal (Simmons, Gordon, \& Chambless, 2005; Seider, Hirschberger, Nelson, \& Levenson, 2009). Segundo os pesquisadores, o emprego dos pronomes revela informações sobre a qualidade da interação e do vínculo entre os cônjuges, sobre a expressão e regulação das emoções e sobre como os problemas são resolvidos. Os pronomes são divididos em integradores e de separação. Os integradores, eu e nós, produzem soluções mais positivas para os conflitos e implicam no trabalho conjunto entre os parceiros, mesmo quando a conversa está centrada em uma área conflituosa. $\mathrm{O}$ pronome de separação, tu/você, remete a um sentido maior de independência e distanciamento entre os cônjuges e está associado à insatisfação conjugal e às interações mais negativas durante as situações de conflito.

Finalmente, percepções, necessidades, expectativas e comportamentos sofrem alterações ao longo do tempo de casamento, possivelmente, associadas à etapa do ciclo vital do casal e ao amadurecimento individual e da própria dinâmica conjugal (Bertoni \& Bodenmann, 2010; Hahlweg \& Richter, 2010; Silva \& Vandenberghe, 2009). Tal pressuposto indica que as estratégias de resolução de conflito podem mudar (Hoppmann \& Blanchard-Fields, 2011; Smith et al., 2009; Stieglitz, Gurven, Kaplan, \& Winking, 2012). Por esse motivo, e considerando que a presente investigação enfocou casamentos de longa duração, avaliar o tempo de relacionamento foi fundamental. Como parâmetro para esta variável, foram consideradas relações conjugais de longa duração aquelas com tempo de união superior à média dos casamentos oficiais no Brasil que, segundo dados do IBGE (2013), são de 15 anos.

Diante do exposto, percebe-se que estratégias construtivas e destrutivas coexistem e podem mudar ao longo do tempo de união marital. Nesse sentido, como prevenir estratégias destrutivas no casamento? Segundo Gottman (1998) e Johnson et al. (2005), mudar padrões de interação pode alterar as estratégias de resolução dos conflitos. Whiting (2008) defende mudanças no comportamento, na percepção do outro e da relação, nas atitudes, no compromisso com o parceiro e no senso de responsabilidade pessoal sobre os problemas. Outros estudos apontam que o casal precisa dispor de um espaço para comunicar suas dificuldades e queixas, 
mudar questionamentos e percepções sobre os conflitos e identificar aspectos positivos (Fincham et al., 2004; Fincham et al., 2007; Paleari et al., 2010; Rasera \& Guanaes, 2010; Veldorale-Brogan et al., 2013).

Além disso, resolver os problemas por meio de estratégias construtivas repercute positivamente na conjugalidade através de maiores níveis de qualidade e satisfação (Mosmann, Wagner, \& Sarriera, 2008), reforça a aliança entre os cônjuges e os fortalece diante do estresse diário (Anderson \& Johnson, 2010). Por outro lado, utilizar estratégias destrutivas resulta em menores níveis de satisfação conjugal, provoca sentimentos de mágoa e de angústia e pode culminar em separação (Paleari et al., 2010; Zordan, Wagner, \& Mosmann, 2012).

As estratégias de resolução dos conflitos conjugais têm sido expressivamente investigadas no contexto internacional, porém, há significativa carência de estudos nacionais sobre o tema (Bolze, Crepaldi, Schmidt, \& Vieira, 2013). Ademais, é necessário investigar a interação entre diferentes estratégias, construtivas e destrutivas, e variáveis como, tipos de problema, características dos parceiros, padrões de interação e tempo de casamento. Dessa forma, o objetivo nessa pesquisa foi identificar e compreender as estratégias de resolução de conflito utilizadas em casamentos de longa duração e a sua reverberação na dinâmica conjugal.

\section{MÉTODO}

\section{Delineamento}

Trata-se de uma pesquisa qualitativa de caráter exploratório e descritivo. Foram analisadas experiências e significações que emergiram da interação entre os participantes do grupo focal com o objetivo de conhecer o fenômeno, identificar relações existentes entre os elementos que o compõem, obter novas percepções e descrever as características encontradas (Gibbs, 2009; Turato, 2008).

\section{Participantes}

Os integrantes do grupo focal foram selecionados de uma amostra de 200 pessoas que participaram de um estudo maior no Núcleo de Estudos de Casal e Família - NECAF. Os respondentes que marcaram, no final do instrumento, a opção "tenho interesse em continuar participante de pesquisas no NECAF" foram contatados. O grupo foi constituído por nove pessoas: cinco mulheres e quatro homens, heterossexuais, vivendo em união estável, porém, não unidos maritalmente entre si no grupo focal, com filhos e residentes em Porto Alegre e região metropolitana.
A idade mínima dos participantes foi de 40 anos e a máxima de 57 anos; o tempo de união variou de 20 a 32 anos; quanto à escolaridade, quatro participantes cursaram ensino médio ou técnico e cinco o ensino superior; todos exerciam atividade remunerada, trabalhando de seis a 14 horas/dia; a remuneração pelo trabalho variou de 2 e 4 salários mínimos $(n=4)$, de 6 e $10(n=2)$ e de 10 e 15 salários $(n=3)$; o número de filhos foi de 1 a 4, sendo que a idade da prole variou de 2 a 31 anos de idade.

\section{Instrumento}

$\mathrm{O}$ instrumento utilizado foi o grupo focal que tem como característica ser pequeno e ocorrer em ambiente não diretivo onde são discutidos temas específicos. Há influência mútua entre os participantes por meio da interação, propiciando a emersão de questões individuais e coletivas enriquecedoras do material de análise. Os participantes são selecionados de acordo com o objetivo do estudo que determinará as características do grupo (Flick, 2009; Minayo, Souza, Constantino, \& Santos, 2008). O encontro realizado nessa pesquisa teve a seguinte estrutura: (a) Acolhimento - recepção dos participantes pelos moderadores; (b) Abertura - apresentação dos moderadores, assinatura do TCLE, informações gerais sobre os objetivos do encontro e sobre como ele ocorreria; (c) Aquecimento - apresentação dos participantes e debate sobre características comuns aos relacionamentos amorosos; (d) Discussões Temáticas - utilização de questões disparadoras pelos moderadores, tais como: - O que se espera de um casamento? - Quais os motivos de conflito entre os cônjuges? - Sentimentos durante os episódios de conflito? (e) Encerramento. Ressalta que os moderadores estiveram atentos para que todos os integrantes do grupo expusessem seus pontos de vista e interagissem durante os debates (Flick, 2009).

\section{Coleta de dados}

Foram contatadas 38 pessoas por telefone aproximadamente um ano depois de terem participado do estudo maior. Destas, 17 declinaram do convite, 21 confirmaram participação e 9 comparecem no dia agendado para a realização do grupo focal. Na ligação telefônica, os participantes foram convidados para um encontro em que se debateriam temas relacionados ao cotidiano da vida conjugal, como os motivos de conflito no casamento e as estratégias utilizadas para resolvêlos. Dois moderadores, psicólogos e terapeutas de casal e família, conduziram o grupo focal que aconteceu em espaço reservado na instituição de ensino à qual o projeto maior estava vinculado. $\mathrm{O}$ encontro iniciou às $19 \mathrm{~h} 30 \mathrm{~min}$. e terminou às $22 \mathrm{~h}$. 


\section{Análise dos dados}

As conversações no grupo focal, ou seja, o diálogo entre os participantes, foram transcritas integralmente e examinadas, por ambos moderadores através do método de análise de conteúdo, em que se aplicam procedimentos sistemáticos e objetivos de descrição dos conteúdos e são feitas inferências válidas no texto analisado (Bauer, 2008). As etapas da análise de conteúdo foram: a) Leitura repetida do material transcrito com o objetivo de conhecer integralmente o texto; b) Identificação das unidades de sentido; c) Organização das unidades de sentido em categorias temáticas definidas a posteriori, e apresentadas em dois eixos; d) Identificação de intenções particulares, considerando o conteúdo manifesto, a interpretação e as inferências com base na experiência do pesquisador e à luz da teoria sistêmica.

\section{Procedimentos éticos}

O estudo foi submetido ao Comitê de Ética em Pesquisa da Instituição de Ensino e aprovado sob o parecer $n$ o 495.313. O TCLE, lido e assinado pelos participantes, informou que o encontro seria gravado em áudio e vídeo, entretanto, seria preservada a identidade dos membros do grupo. Os integrantes do grupo foram informados que poderiam desistir de participar antes de assinar o TCLE sem nenhum prejuízo e, ainda, que o risco que o encontro oferecia era comoção frente a algum tema ou discussão sendo, nestes casos, avaliada a situação e realizados os encaminhamentos necessários. Além disso, foram seguidas todas as diretrizes e normas regulamentadoras de pesquisas envolvendo seres humanos, conforme orientações das Resoluções 466/2012 do Conselho Nacional de Saúde (2012) e 016/2000 do Conselho Federal de Psicologia (2000).

\section{RESULTADOS E DISCUSSÃO}

Por meio da análise de conteúdo emergiram cinco categorias temáticas que foram agrupadas em dois eixos. No eixo um "Estratégias de resolução dos conflitos conjugais" são apresentadas três categorias: a) estratégias de resolução construtivas; b) estratégias de resolução destrutivas e c) comportamentos que interferem nas estratégias de resolução dos conflitos. No eixo dois "Através do tempo: características individuais e conjugais", são apresentadas duas categorias: a) características individuais estáticas e dinâmicas dos cônjuges e b) padrões de interação entre os cônjuges durante a resolução dos conflitos. As categorias são exemplificadas apenas com as falas mais representativas do conteúdo exposto. Para preservar a identidade dos participantes, seus nomes foram substituídos por códigos, quais sejam: homens como H1, H2, H3 e H4 e mulheres como M1, M2, M3, M4 e M5.

\section{Estratégias de resolução dos conflitos conjugais}

$\mathrm{Na}$ primeira categoria do eixo um "estratégias de resolução construtivas", os participantes referiram que as estratégias que funcionam construtivamente diante dos conflitos são: conversar pacificamente; manter a calma e se controlar; avaliar as consequências do que será dito ao parceiro, especialmente, no momento da raiva. Ainda, escrever por meio de carta, Skype ou mensagem de texto no celular, ao invés de falar, pois permite filtrar o conteúdo e repensar o que será dito; colocar-se no lugar do outro nas situações de conflito; perceber a hora de recuar; ter postura ativa diante dos conflitos, respeitar o parceiro, entre outras. Algumas das estratégias mencionadas aparecem na seguinte parte da discussão:

"O que resolve é uma boa conversa né. Diálogo." (H-4)

"Dialogar e as vezes quando tu tá com muita raiva, eu pego e escrevo, já mandei cartas pro meu marido dentro de casa. Ainda bem que eu rasguei algumas né, porque eu escrevi tanta bobagem, eu escrevi tanta coisa, porque eu estava com tanta raiva." (M-1)

"Eu já resolvi alguns conflitos com a minha esposa até mesmo por mensagem. Já fizemos até comunicação por msn. Eu conversava com ela, dizia, olha, eu sei que pisei na bola. Tu errou comigo e tal. A gente ia se comunicando e era muito mais fácil, dessa vez foi muito mais fácil de se colocar as coisas." (H-1)

Estes resultados corroboram a literatura sobre as principais estratégias construtivas de resolução dos conflitos (Anderson \& Johnson, 2010; Falcke et al., 2013; Rasera \& Guanaes, 2010; Sierau \& Herzberg, 2012; Sullivan et al., 2010; Wheeler et al., 2010; Whiting, 2008). Além disso, a capacidade de dialogar, a demonstração de abertura para resolver o problema e o reconhecimento dos próprios erros estão associados, respectivamente, aos três fatores que constituem as estratégias de resolução dos conflitos: comunicação, virtude e identidade, conforme proposto por VeldoraleBrogan et al. (2013). 
Considerando que a média de tempo de união dos participantes do grupo foi de 27 anos, tais resultados corroboram os achados de Hoppmann e BlanchardFields (2011) sobre casais mais velhos utilizarem estratégias de colaboração e, também, outros autores (Seider et al., 2009; Simmons et al., 2005) sobre a corresponsabilidade pelo relacionamento, pelas dificuldades e pelos conflitos que existem entre o casal. Conjectura-se que a maior parte das estratégias construtivas referidas estejam relacionadas à capacidade de ouvir o parceiro e colaborar para a resolução dos problemas. Este resultado pode ser entendido como um conjunto de fatores individuais e diádicos que interagem sistemicamente e, juntos, produzem efeitos mais positivos ou negativos, dependendo de como essa interação acontece.

Foi compreendida, enquanto estratégia construtiva de resolução dos conflitos, a tentativa de identificar o momento em que o cônjuge demonstra estar receptivo para conversar sobre os problemas:

"Eu fico as vezes dois, três dias, esperando um momento pra nós né, e que ela possa estar receptiva a uma conversa, não uma discussão." (H-4)

"Eu acho que a gente tem que perceber, e eu aprendi isso a duras penas, perceber o outro, no sentido assim, agora é o momento que eu posso falar." (M-3)

Este resultado pode indicar que avaliar o clima conjugal e o estado emocional do parceiro (Sullivan et al., 2010) é uma possível estratégia construtiva de resolução dos conflitos. Pode apontar também que a conexão emocional entre os cônjuges, a empatia e a auto revelação são efetivamente relevantes diante dos problemas conjugais (Hoppmann \& Blanchard-Fields, 2011; Leggett et al., 2012; Paleari et al., 2010; Tallman \& Hsiao, 2004).

Ainda com relação ao momento mais indicado para tentar resolver os conflitos, o grupo foi questionado sobre a percepção de si mesmo durante os episódios de conflito:

"Era difícil eu dar o braço a torcer. Agora já não é mais tanto, mas eu era teimoso demais bah." (H-2)

"Principalmente no momento em que a raiva já passou. Eu sempre me controlei muito. Minha irmã dizia assim pra mim, M3 tu pensa demais, vai lá e fala logo. E eu dizia, não dá, eu não consigo, não é a minha hora, eu tenho que sentir a hora." (M-3)
O resultado corrobora outros estudos (Cundiff et al., 2012; Hoppmann \& Blanchard-Fields, 2011; Veldorale-Brogan et al., 2013) sobre o processamento individual que se faz da informação antes, durante e após a ocorrência do conflito. Nesse caso, sentimentos e pensamentos são avaliados, prevendo que equívocos repercutirão negativamente ao se abordar o problema, isto é, o indivíduo avalia as possíveis reações que terá diante da situação de conflito antes de conversar com o parceiro.

De acordo com a discussão realizada no grupo focal, diferentes posicionamentos sobre o momento oportuno para tentar resolver os conflitos foram identificados:

"A gente nunca deixa pra amanhã se não a briga gera dois dias." (M-2)

"Eu deixava baixar a poeira, dormia, porque senão muita coisa tu vai falar que não precisa, até pra ti também poder se acalmar e ver o outro lado." (M-5)

As diferentes opiniões, se o mais indicado é resolver o conflito na hora/dia em que ocorre, identificar o momento certo ou adiar a resolução, podem indicar como estratégia construtiva avaliar o motivo e a gravidade do conflito (McNulty et al., 2008; McNulty \& Russell, 2010). Além disso, pode sugerir que a expectativa e a necessidade de resolução do problema, no mesmo dia ou não, estão associadas às características de cada pessoa e à interpretação que faz da situação (Cundiff et al., 2012; Madhyastha et al., 2011).

Na segunda categoria do eixo um são apresentadas as "estratégias de resolução destrutivas". Foram identificados comportamentos de retaliação e a repercussão destrutiva que provocam entre os parceiros, conforme aponta a literatura (Fincham et al., 2007; McNulty, et al., 2008; Paleari et al., 2010).

"Meu marido um dia bebeu e começou a me dizer um monte de coisa, ele me chamou de gorda, ai eu enfureci. Eu disse, ah tu precisa beber pra me ofender, pois então eu vou fazer a mesma coisa que tu. Mas eu disse tudo que eu tinha vontade pra ele, bêbada também né, e não diz que bêbado esquece, porque bêbado não esquece. Eu me lembro de tudo que eu falei." (M-2)

Apesar de a situação tornar evidente padrões de interação disfuncionais, percebe-se que a estratégia encontrada por M2 para rebater as ofensas do marido e lidar com a situação foi revidar por meio de 
comportamentos de retaliação (McNulty et al., 2008; McNulty \& Russell, 2010). Outros participantes do grupo referiram como destrutivo: ser impulsivo, irritarse, lidar com os problemas de forma excessivamente racional, impor que o outro mude seu jeito de ser, tentar adivinhar o que o cônjuge está pensando ou sentindo, desistir da situação ou evitar resolver determinados conflitos.

“Tu não pode desistir de uma situação, né. É que nem tu pegar a sujeira e jogar ela para baixo do tapete. Não! tu tem que enfrentar aquela situação." (H-1)

"A minha experiência é de que enquanto a gente quer tentar mudar o pensamento dele, não, eu não consegui nada. No início, eu queria que ele fosse do meu jeito, que ele fizesse as coisas, então eu passei a aceitar isso e tipo a usar uma outra estratégia." (M-3)

Esses resultados são evidências de que os integrantes do grupo possuem clareza das estratégias funcionais e daquelas destrutivas para eles, corroborando outros estudos quanto às estratégias destrutivas (Anderson \& Johnson, 2010; Falcke et al., 2013; Rasera \& Guanaes, 2010; Sierau \& Herzberg, 2012; Sullivan et al., 2010; Wheeler et al., 2010; Whiting, 2008). Congruente aos pressupostos de alguns pesquisadores, os participantes referiram que determinados conflitos podem ser resolvidos na hora, enquanto outros não. Para tanto, seria necessário avaliar o motivo/área e a gravidade do conflito (Hoppmann \& Blanchard-Fields, 2011; Verhofstadt et al., 2005), o estado emocional do cônjuge (Sullivan et al., 2010) e a interpretação que cada membro do casal faz do problema (Cundiff et al., 2012; Madhyastha et al., 2011).

Além disso, pensar sobre o que será dito durante o conflito, segundo os participantes, pode evitar mágoas e arrependimento. Porém, tal postura não deve representar excesso de submissão, em que se aceitam quaisquer posicionamentos ou se assume a responsabilidade integral pelos problemas (Smith et al., 2009). Compreende-se, a partir desses resultados, que há uma diferença tênue entre o desfecho mais positivo ou mais negativo de um conflito, corroborando outras pesquisas (Falcke et al., 2013; Wheeler et al., 2010) em que se defende ser esperado que os parceiros oscilem entre estratégias construtivas e destrutivas ao longo do casamento.

$\mathrm{Na}$ última categoria do eixo um foram identificados "comportamentos que interferem nas estratégias de resolução dos conflitos". Os comportamentos encontrados foram: egoísmo, arrogância, orgulho e inflexão. Por outro lado, o grupo discutiu que com o tempo os cônjuges vão ficando mais tolerantes, compreensíveis e flexíveis, desenvolvem a capacidade de respeitar o jeito de ser e a opinião do parceiro sobre as diversas questões que envolvem a vida conjugal e familiar.

"É ai que entra a compreensão né. Vamos criar atrito por isso? Eu tive que aceitar, criar o meu nivel de aceitação né, pra o meu nivel de tolerância aumentar." (H-4)

"Eu acho que aquilo que move a questão mesmo, seja que conflito for, é sempre o nosso egoísmo, não só do outro, o nosso egoísmo. Porque a gente tem uma tendência assim de querer que as coisas sejam do nosso jeito, sejam como eu acho que é certo." (M-3)

Segundo os participantes do grupo, ao longo do tempo de união, os parceiros aperfeiçoam sua capacidade de reconhecer as próprias fraquezas, de interpretar as situações de conflito, de compreender e de gerenciar os problemas presentes no casamento. Esses fatores podem significar efetivamente uma mudança de postura diante dos problemas conjugais, confirmando o estudo de Schoebi et al. (2012) de que comportamentos de mudança reverberam positivamente na conjugalidade a longo prazo, proporcionando estabilidade conjugal, maiores níveis de satisfação no relacionamento e prevenindo a separação.

\section{Através do tempo: características individuais e conjugais}

Na primeira categoria do eixo dois são apresentadas as "características individuais estáticas e dinâmicas dos cônjuges". A partir das discussões no grupo, compreende-se que existem características individuais e determinadas situações no relacionamento que, muitas vezes, assumem um caráter definitivo.

"Não vamos nem discutir porque não vai valer a pena. O que não tem solução, solucionado está, esquece, coloca uma pedra em cima." (H-2)

"Só que isso, isso faz trinta anos e eu não consegui mudar, entendeu." [referindo-se a uma característica da esposa] $(\mathrm{H}-3)$

"Eu acho que isso depende do perfil de cada um, de cada pessoa, da personalidade também, porque se a pessoa já tem essa tendência mais meiga, de 
abrir mão assim da postura dela, de se malear né. Já não é o meu caso, eu sou impositiva, eu sou mandona. Eu não estou criticando, eu acho que paciência é uma virtude que eu tô trabalhando. Talvez o correto seria um meio termo, mas eu não sou assim." (M-1)

Os participantes referiram aceitar, ao longo do tempo de união, que certas características do parceiro e da dinâmica conjugal não iriam mudar. Segundo eles, por meio da aceitação, tais situações deixaram de ser motivo de discórdia no casamento. Entretanto, foi referido que tal postura não significa desistir dos problemas conjugais por dificuldades de iniciar movimentos de mudança (Cundiff et al., 2012; Madhyastha et al., 2011), mas ter clareza de que nem todos os problemas existentes em um relacionamento amoroso serão resolvidos.

Além disso, alguns comportamentos referidos no grupo pareceram habilidades desenvolvidas ao longo do tempo de relacionamento, ou que existiam, mas foram sendo aperfeiçoadas (Bertoni \& Bodenmann, 2010). Os participantes referiram: auto controle, clareza sobre as limitações existentes em um casamento, expectativas reais em torno da conjugalidade e flexibilidade.

"Eu era explosiva, já falava tudo, agora tu já vai vendo." (M-5)

"Eu acho que tem uma fase do teu relacionamento conjugal que a gente começa a ceder. A gente tem que conviver com as diferenças, tem coisas que a gente muda e tem outras coisas que só o tempo. Vai passando o tempo, a gente vai amadurecendo, vai conseguindo o auto controle também, controlar essas emoções." (H-1)

"É uma aceitação da pessoa como ela é. Houve uma aceitação dele, ao longo do tempo obviamente né, teve todo um espaço de tempo ali que se pisou em ovos e aquela coisa toda, mas daqui um pouco a coisa foi se encaixando, daqui a pouco ele até me dava força, daqui um pouco ele até me incentivava." (M-3)

Esses resultados provocam reflexões sobre os fatores associados às mudanças que, segundo o grupo, ocorreram ao longo do casamento. Conjecturam-se três possibilidades: a) os cônjuges realmente conseguiram diminuir áreas de tensão e conflito por meio de estratégias construtivas, b) avaliaram e aceitaram que determinadas características e situações não mudam, ou c) renderam-se frente ao esforço de tentar resolver problemas que pareciam ser difíceis, seja por cansaço ou por comodismo.

Essas hipóteses confirmam os pressupostos de que mudar é efetivamente difícil. Porém, se a tentativa for bem-sucedida, repercutirá positivamente na conjugalidade a longo prazo (Madhyastha et al., 2011; Schoebi et al., 2012; Sierau \& Herzberg, 2012). Na terceira hipótese, os cônjuges permanecem na relação, porém, não há investimento e envolvimento em comportamentos efetivos de mudança, mas apenas o desejo de permanecer casado, situação apontada por Schoebi et al. (2012) como deletéria à conjugalidade.

A segunda categoria do eixo dois foi denominada "padrões de interação entre os cônjuges durante a resolução dos conflitos". Os participantes falaram sobre contextos de interação conjugal e sobre o que pensam de determinados padrões de interação:

"Talvez ela possa conviver com isso tranquilamente [referindo-se à participante que disse não tocar em assuntos que porventura possam magoar o cônjuge], mas nós, lá em casa, não convivemos dessa forma, na nossa personalidade, é o nosso caso." (H-1)

"Tu fala daquilo que tu pode fazer, porque sempre mudar o outro né. O outro pode até mudar em consequência das tuas atitudes. Ele vai mudar a postura dele, mas não que o teu querer, a tua vontade, seja a do outro." (M-3)

Essa categoria e a anterior são complementares, uma vez que a interferência das características individuais, durante a interação conjugal, provoca um padrão relacional através da influência que um membro da relação exerce sobre o outro. Além disso, os resultados dessa categoria diferem do que foi apontado por Madhyastha et al. (2011) pois sinalizam que a resolução dos conflitos dependerá também dos níveis de negatividade e de positividade que podem emergir durante o conflito. Desse modo, os padrões de interação se retroalimentam por meio da contribuição individual de cada cônjuge, corroborando Cundiff et al. (2012) sobre o efeito sócio, em que os membros do casal se influenciam mutuamente.

Nessa perspectiva, as características individuais diante dos conflitos e das tentativas de resolvê-los, atualizam-se durante a interação entre os cônjuges e podem ser compreendidas enquanto dinâmica do casal. Além disso, a variável tempo de casamento pode estar associada ao resultado encontrado, visto que, ao longo dos anos, o indivíduo passa por experiências pessoais e conjugais que alteram suas concepções de mundo, seus 
valores e, consequentemente, os padrões de interação entre os cônjuges, constituindo uma complexa rede de interações que (se) atualizam constantemente a (na) dinâmica conjugal.

\section{CONSIDERAÇÕES FINAIS}

Entre os resultados e contribuições desse estudo, a atenção sobre a conjugalidade emergiu como uma possível estratégia de prevenção dos conflitos. Tratase de estar atento às próprias emoções e reações; à predisposição do parceiro ao diálogo e a aproximação; ao clima conjugal e aos motivos e níveis de conflito, se mais ou menos graves, passíveis ou não de mudança. Essa "estratégia" pode ser uma forma de evitar que os problemas fiquem mais difíceis de resolver, visto que preventivamente os cônjuges tomariam o cuidado de avaliar os critérios citados e que, neste estudo, emergiram como relevantes à resolução dos conflitos.

Além disso, foram identificadas características dinâmicas, que mudam, ao longo do tempo de casamento, associadas ao investimento dos parceiros e às alterações que ocorrem no indivíduo e nos padrões de interação. Entretanto, emergiram também características estáticas, apontando peculiaridades do relacionamento e do jeito de ser dos cônjuges dificilmente passíveis de mudança. Se a decisão for permanecer na relação apesar das características e padrões de interação estáticos, investir nos aspectos positivos do parceiro e do relacionamento, reservar momentos de intimidade exclusivos ao casal e se unir frente aos conflitos do dia-a-dia podem ser estratégias que contribuem para minimizar as áreas de tensão no casamento.

Revelou-se importante também refletir quanto às expectativas dentro do relacionamento em três níveis: a) sobre si mesmo e o papel exercido na relação, avaliando a responsabilidade sobre os conflitos e os movimentos realizados para que as mudanças esperadas ocorram; b) sobre o parceiro, de que corresponda à prérequisitos e se dedique exclusivamente ao casamento, e c) sobre a conjugalidade, de que proporcione satisfação em tempo integral e não tenha conflitos. Entre essas e outras possíveis expectativas presentes em cada nível é fundamental que os cônjuges avaliem se são reais e possíveis de alcançar.

Pode ser uma limitação deste estudo, ter analisado conflitos e estratégias aparentemente bem-sucedidas, de modo que o discurso está, em certa medida, elaborado. Acontece que o número de pessoas que concordam em contribuir com as pesquisas, conforme descrito no item "coleta de dados", é bastante reduzido. Geralmente, as pessoas que participam de estudos científicos apresentam baixos níveis de conflito no casamento, enquanto casais que enfrentam dificuldades mais acentuadas, tendem a declinar dos convites.

Por outro lado, indivíduos em casamentos de longa duração debateram sobre conflitos conjugais, estratégias e mudanças que ocorreram ao longo de todo um percurso conjugal, o que possibilitou ampliar as discussões sobre o tema proposto. Além disso, as variáveis que, neste estudo, contribuíram para a compreensão da reverberação das estratégias de resolução na dinâmica conjugal, características individuais, tempo de casamento e padrões de interação, podem ser analisadas, em futuras pesquisas com método quantitativo, verificando se e como predizem a forma como os casais resolvem seus conflitos.

O estudo explorou as estratégias de resolução dos conflitos conjugais, citadas na literatura nacional e internacional, corroborando a maior parte dos resultados encontrados nestas pesquisas. Possibilitou compreender também que as diferentes estratégias e a reverberação que provocam na dinâmica conjugal estão associadas a outras variáveis que precisam ser melhor investigadas. Para tanto, recomenda-se que estudos por meio de delineamento explicativo sejam realizados, para que no contexto nacional, seja ampliado e aprofundado o conhecimento científico sobre as variáveis associadas mais fortemente ao fenômeno investigado nessa pesquisa.

\section{REFERÊNCIAS}

Anderson, S., \& Johnson, L. (2010). A dyadic analysis of the between and within system alliances on distress. Family Process, 49, 220-235. http://dx.doi.org/10.1111/j.1545-5300.2010.01319.x

Bauer, M. W. (2008). Análise de conteúdo clássica: Uma revisão. In M. W. Bauer, \& G. Gaskell. Pesquisa qualitativa com texto, imagem e som: um manual prático (P. A. Guareschi, trad., 7. ed., pp. 189-217). Petrópolis, RJ: Vozes.

Bertoni, A., \& Bodenmann G. (2010). Satisfied and dissatisfied couples: Positive and negative dimensions, conflict styles, and relationships with family of origin. European Psychologist, 15, 175-184. http://dx.doi.org/10.1027/1016-9040/ a000015

Bolze, S. D. A., Crepaldi, M. A., Schmidt, B., \& Vieira, M. L. (2013). Relacionamento conjugal e táticas de resolução de conflito entre casais. Actualidades en Psicología, 27(114), 71-85. Recuperado em: 17 fev. 2014, de <http://kerwa. ucr.ac.cr/handle/10669/8892>. http://dx.doi.org/10.15517/ap.v27i114.4828 
Carlson, J., \& Dinkmeyer, D. (1987). Adlerian marriage therapy. The American Journal of Family Therapy, 15, $326-332$. http://dx.doi.org/10.1080/01926188708250692

Cundiff, J. M., Smith, T. W., \& Frandsen, C. A. (2012). Incremental validity of spouse ratings versus self-reports of personality as predictors of marital quality and behavior during marital conflict. Psychological Assessment, 24, 676-684. http://dx.doi.org/10.1037/a0026637

Falcke, D., Wagner, A., \& Mosmann, C. (2013). Estratégias de resolução de conflito e violência conjugal. In T. FéresCarneiro (Eds.), Casal e Família: transmissão, conflito e violência (pp. 159-176). São Paulo: Caso do Psicólogo.

Fincham, F. D., Beach, S. R., \& Davilla, J. (2004). Forgiveness and conflict resolution in marriage. Journal of Family Psychology, 18, 72-81. http://dx.doi.org/10.1037/0893-3200.18.1.72

Fincham, F. D., Beach, S. R., \& Davila, J. (2007). Longitudinal relations between forgiveness and conflict resolution in marriage. Journal of Family Psychology, 21, 542-545. http://dx.doi.org/10.1037/0893-3200.21.3.542

Flick, U. (2009). Introdução à pesquisa qualitativa (3a ed.). Porto Alegre: Artmed.

Gibbs, G. (2009). Análise de dados qualitativos. Porto Alegre: Artmed.

Gottman, J. M. (1998). Psychology and the study of marital processes. Annual Review of Psychology, 49, 169-197. http:// dx.doi.org/10.1146/annurev.psych.49.1.169

Hahlweg, K., \& Richter, D. (2010). Prevention of marital instability and distress: Results of an 11-year longitudinal follow-up study. Behaviour Research and Therapy, 48, 377-383. http://dx.doi.org/10.1016/j.brat.2009.12.010

Hoppmann, C. A., \& Blanchard-Fields, F. (2011). Problem-solving variability in older spouses: How is it linked to problem, person, and couple-characteristics? Psychology and Aging, 26, 525-531. http://dx.doi.org/10.1037/ a0024114

Instituto Brasileiro de Geografia e Estatística [IBGE]. (2013). Estatística de Registro Civil (Vol. 40). Rio de Janeiro: Autor. Recuperado em 22 maio 2014, de <http://biblioteca.ibge.gov.br/visualizacao/periodicos/135/rc_2013_v40. pdf $>$.

Johnson, M. D., Cohan, C. L., Davila, J., Lawrence, E., Rogge, R. D., Karney, B. R., Bradbury, T. N. et al. (2005). Problem-solving skills and affective expressions as predictors of change in marital satisfaction. Journal of Consulting and Clinical Psychology, 73, 15-27. http://dx.doi.org/10.1037/0022-006X.73.1.15

Leggett, D. G., Roberts-Pittman, B., Byczek, S., \& Morse, D. T. (2012). Cooperation, conflict, and marital satisfaction: Bridging theory, research, and practice. The Journal of Individual Psydiology, 68(2), 182-199. Recuperado em 17 fev. 2014, de <http://connection.ebscohost.com/c/articles/76259603/cooperation-conflict-marital-satisfaction-bridgingtheory-research-practice>.

Madhyastha, T. M., Hamaker, E. L., \& Gottman, J. M. (2011). Investigating spousal influence using moment-to-moment affect data from marital conflict. Journal of Family Psychology, 25, 292-300. http://dx.doi.org/10.1037/a0023028

McNulty, J. K., O’Mara, E. M., \& Karney, B. R. (2008). Benevolent cognitions as a strategy of relationship maintenance: "Don't sweat the small stuff" ... but it is not all small stuff. Journal of Personality and Social Psychology, 94, 631-646. http://dx.doi.org/10.1037/0022-3514.94.4.631

McNulty, J. K. \& Russell, V. M. (2010). When "negative" behaviors are positive: A contextual analysis of the longterm effects of problem-solving behaviors on changes in relationship satisfaction. Journal of Personality and Social Psychology, 98, 587-604. http://dx.doi.org/10.1037/a0017479

Minayo, M. C. S., Souza, E. R., Constantino, P., \& Santos, N. C. (2008). Métodos, técnicas e relações em triangulação. In M. C. S. Minayo, S. G. Assis, \& E. R. Souza (Orgs.), Avaliação por triangulação de métodos: abordagem de programas sociais (20 ed.) (pp. 71-103). Rio de Janeiro: Fiocruz.

Mosmann, C.\& Falcke, D. (2011). Conflitos conjugais: Motivos e frequência. Revista da SPAGESP, 12(2), 5-16. Recuperado em 18 fev. 2014, de <http://pepsic.bvsalud.org/scielo.php?script=sci_arttext\&pid=S1677-29702011000200002>.

Mosmann, C. P., Wagner, A., \& Sarriera, J. (2008). A qualidade conjugal como preditora dos estilos educativos parentais: O perfil discriminante de casais com filhos adolescentes. Revista da Associação Portuguesa de Psicologia, 22(2), 161-182. Recuperado em 17 fev. 2014, de <http://revista.appsicologia.org/index.php/rpsicologia/article/view/352>.

Paleari, F. G., Regalia, C., \& Fincham, F. D. (2010). Forgiveness and conflict resolution in close relationships: Within and cross partner effects. Universitas Psychologica, 9(1), 35-56. Recuperado em 17 fev. 2014, de <http://www.redalyc. org/articulo.oa?id=64712156004>.

Rasera, E. F. \& Guanaes, C. (2010). Momentos marcantes na construção da mudança em terapia familiar. Psicologia: Teoria e Pesquisa, 26(2), 315-322. Recuperado em 18 fev. 2014, de <https://revistaptp.unb.br/index.php/ptp/article/ view/383>.

Schoebi, D., Karney, B. R., \& Bradbury, T. N. (2012). Stability and change in the first 10 years of marriage: Does commitment confer benefits beyond the effects of satisfaction? Journal of Personality and Social Psychology, 102, 729-742. http://dx.doi.org/10.1037/a0026290

Seider, B. H., Hirschberger, G., Nelson, K. L., \& Levenson, R. W. (2009). We can work it out: Age differences in relational pronouns, physiology, and behavior in marital conflict. Psychology and Aging, 24, 604-613. http://dx.doi. org/10.1037/a0016950

Sierau, S. \& Herzberg, P. Y. (2012). Conflict resolution as a dyadic mediator: Considering the partner perspective on conflict resolution. European Journal of Personality, 26, 221-232. http://dx.doi.org/10.1002/per.828 
Silva, L. P., \& Vandenberghe, L. (2009). Comunicação versus resolução de problemas numa sessão única de terapia comportamental de casal. Revista Brasileira de Terapia Comportamental e Cognitiva, 11(1), 43-60. Recuperado em 17 fev. 2014, de <http://www.usp.br/rbtcc/index.php/RBTCC/article/view/383>.

Simmons, R. A., Gordon, P. C., \& Chambless, D. L. (2005). Pronouns in marital interaction what do "you" and "i" say about marital health? Psychological Science, 16(12), 932-936. Recuperado em 18 fev. 2014, de <http://pss.sagepub. com/content/16/12/932.short>.

Smith, T. W., Berg, C. A., Florsheim, P., Uchino, B. N., Pearce, G., Hawkins, M., Olsen-Cerny, C., et al. (2009). Conflict and collaboration in middle-aged and older couples: I age differences in agency and communion during marital interaction. Psychology and Aging, 24, 259-273. http://dx.doi.org/10.1037/a0015609

Stieglitz, J., Gurven, M., Kaplan, H., \& Winking, J. (2012). Infidelity, jealousy, and wife abuse among Tsimane foragerfarmers: Testing evolutionary hypotheses of marital conflict. Evolution and Human Behavior, 33, 438-448. http:// dx.doi.org/10.1016/j.evolhumbehav.2011.12.006

Sullivan, K. T., Pasch, L. A., Johnson, M. D., \& Bradbury, T. N. (2010). Social support, problem solving, and the longitudinal course of newlywed marriage. Journal of Personality and Social Psychology, 98, 631-644. http://dx.doi. org/10.1037/a0017578

Tallman, I., \& Hsiao, Y. L. (2004). Resources, cooperation, and problem solving in early marriage. Social Psychology Quarterly, 67, 172-188. http://dx.doi.org/10.1177/019027250406700204

Turato, E. R. (2008). Tratado da metodologia da pesquisa clínico-qualitativa: Construção teórico-epistemológica, discussão comparada e aplicação nas áreas da saúde e humanas (5a ed.). Petrópolis, RJ: Vozes.

Veldorale-Brogan, A., Lambert, N. M., Fincham, F. D., \& DeWall, C. N. (2013). The virtue of problem-solving: Perceived partner virtues as predictors of problem-solving efficacy. Personal Relationships, 20, 511-523. http:// dx.doi.org/10.1111/j.1475-6811.2012.01421.x

Verhofstadt, L. L., Buysse, A., Ickes, W., De Clercq, A., \& Peene, O. J. (2005). Conflict and support interactions in marriage: An analysis of couples interactive behavior and on-line cognition. Personal Relationships, 12, 23-42. http:// dx.doi.org/10.1111/j.1350-4126.2005.00100.x

Wheeler, L. A., Updegraff, K. A., \& Thayer, S. M. (2010). Conflict resolution in mexican-origin couples: Culture, gender, and marital quality. Journal of Marriage and Family, 72, 991-1005. http://dx.doi.org/10.1111/j.17413737.2010.00744.x

Whiting, J. B. (2008). The role of appraisal distortion, contempt, and morality in couple conflict: A grounded theory. Journal of Marital and Family Therapy, 34, 44-57. http://dx.doi.org/10.1111/j.1752-0606.2008.00052.x

Zordan, E. P., Wagner, A., \& Mosmann, C. (2012). O perfil de casais que vivenciam divórcios consensuais e litigiosos: Uma análise das demandas judiciais. Psico-USF, 17(2), 185-194. Recuperado em 17 fev. 2014, de <http://www.scielo. br/scielo.php?script=sci arttext\&pid=S1413-82712012000200002>.

Autores:

CRÍSTOFER BATISTA DA COSTA - Mestre e Doutorando em Psicologia Clínica, Universidade do Vale do Rio dos Sinos - UNISINOS. Clarisse Pereira MosmanN - Doutora em Psicologia, Universidade do Vale do Rio dos Sinos - UNISINOS.

Endereço para correspondência:

Crístofer Batista da Costa

Rua Coronel Corte Real, 913, apto. 204 - Petrópolis

CEP 90630-080 Porto Alegre, RS, Brasil

E-mail: cristoferbatistadacosta@gmail.com

Recebido em: 13.05 .15

Aceito em: 08.10.15 